\title{
Symmetrical Bending of Thin Circular Elastic Plates on Equally Spaced Point Supports
}

\author{
A. F. Kirstein and R. M. Woolley \\ Institute for Basic Standards, National Bureau of Standards, Washington, D.C. 20234
}

(August 23, 1966)

\begin{abstract}
A special application of Bassali's solution for transverse flexure of thin elastic plates supported at several points is presented for the case of symmetrical bending. Equations for moments, shearing forces, and stresses are developed which may be useful for design purposes. The experimental results although limited in quantity are in good agreement with the theoretical predictions.
\end{abstract}

Key Words: Circular plates, concentric loading, design, elasticity, experiment, maximum stresses, symmetrical bending, symmetrically distributed load, theory.

\section{Introduction}

The determination of bending moments, twisting moments, and shearing forces in a thin circular elastic plate subjected to symmetrical bending is a problem which is often encountered in the design and analysis of structural elements or systems. This study deals with the solution of this problem for a thin circular elastic plate supported at points equally spaced along a concentric support circle and subjected to a transverse load which is symmetrically distributed over a concentric circular area. These structures may be typified as end closures, bulkheads, and diaphragms. Usually the analysis of such a structure is simplified by the introduction of engineering approximations which pertain to the particular structural system under examination. However, this paper presents a special application of a more general solution developed by Bassali [1] ${ }^{1}$ which more closely represents the conditions realized in practical structures and obviates the necessity for some of the approximations.

This specialized treatment of Bassali's theory provides the equations necessary to calculate moments, shearing forces, and associated stresses anywhere within the plate. It also shows that the expressions for maximum bending stresses at the center are independent of the angular orientation and the number of supports. Further reduction of these expressions result in the Grashof [2] equations.

A comparison between a limited amount of experimental results and the theoretical predictions of tangential strains along the concentric support circle show good agreement. This good agreement for strains along with that for deflections [3] tend to substantiate the theory.

\section{List of Symbols}

$r, \theta$ polar coordinates

$c$ radius of the plate

$a$ radius of the concentric support circle

$b$ radius of the loaded area (region 1) of the plate

$h$ thickness of the plate

' Figures in brackets indicate the literature references at the end of this paper. 


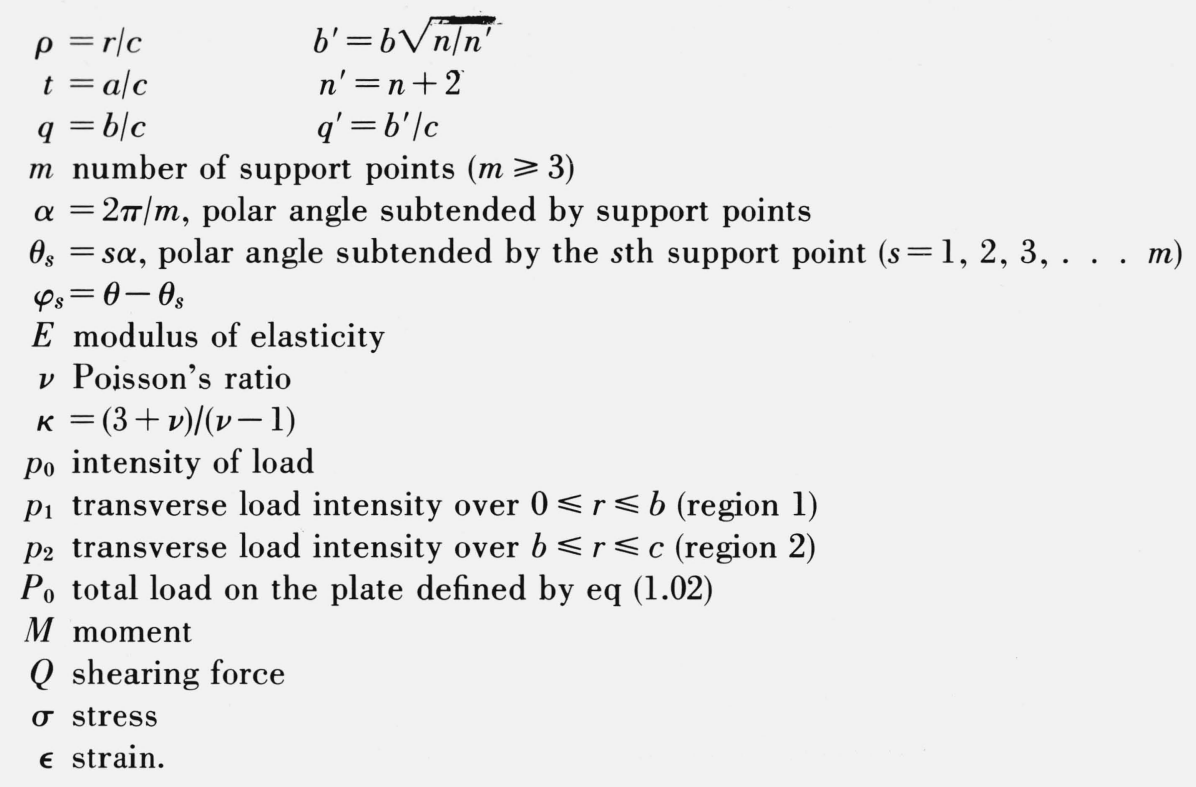

Subscripts:

1,2 refer to regions 1 and 2 , respectively

$r, t$ refer to radial and tangential, respectively

$r t$ refers to twisting.

\section{Background}

Bassali obtained the solution for the problem of flexure of a thin circular elastic plate supported at several arbitrarily located interior points and transversely loaded over a circular area eccentrically located with respect to the center of the plate. Bassali considers the intensity of the transverse load over the circular area indicated as region 1 in figure 1 to be

$$
p_{1}=p_{0} r^{n-2}, \quad(n \geqslant 2)
$$

and the intensity over region 2 to be $p_{2}=0$. This, of course, shows that the load is distributed symmetrically with respect to the center of region 1 , and the value of $n$ defines the load distribution $(n=2$ represents uniform load distribution). The total load on the plate is given by

$$
P_{0}=2 \pi p_{0} \frac{b^{n}}{n}
$$

The boundary of the plate is considered to be free as the plate is supported at interior points, but no special treatment is required when some or all of the supports lie on the boundary.

In a previous paper [3] a special application of this more general solution was used to obtain a method of determining the deflection of a plate subjected to symmetrical bending with the load uniformly distributed over region 1 . A comparison of theoretical and experimental results indicated that the theory adequately predicted the deflection of the plates over the range of geometries tested. Further observations indicated that the theory accounted for a constraining effect on the deflection which for the most part was due to the annular portion of the plate overhanging the support circle. It was also noted that this constraining effect decreased as the number of supports were increased, but did not vanish when the number of supports became sufficiently large to produce a support condition equivalent to that of a simple continuous line support. 


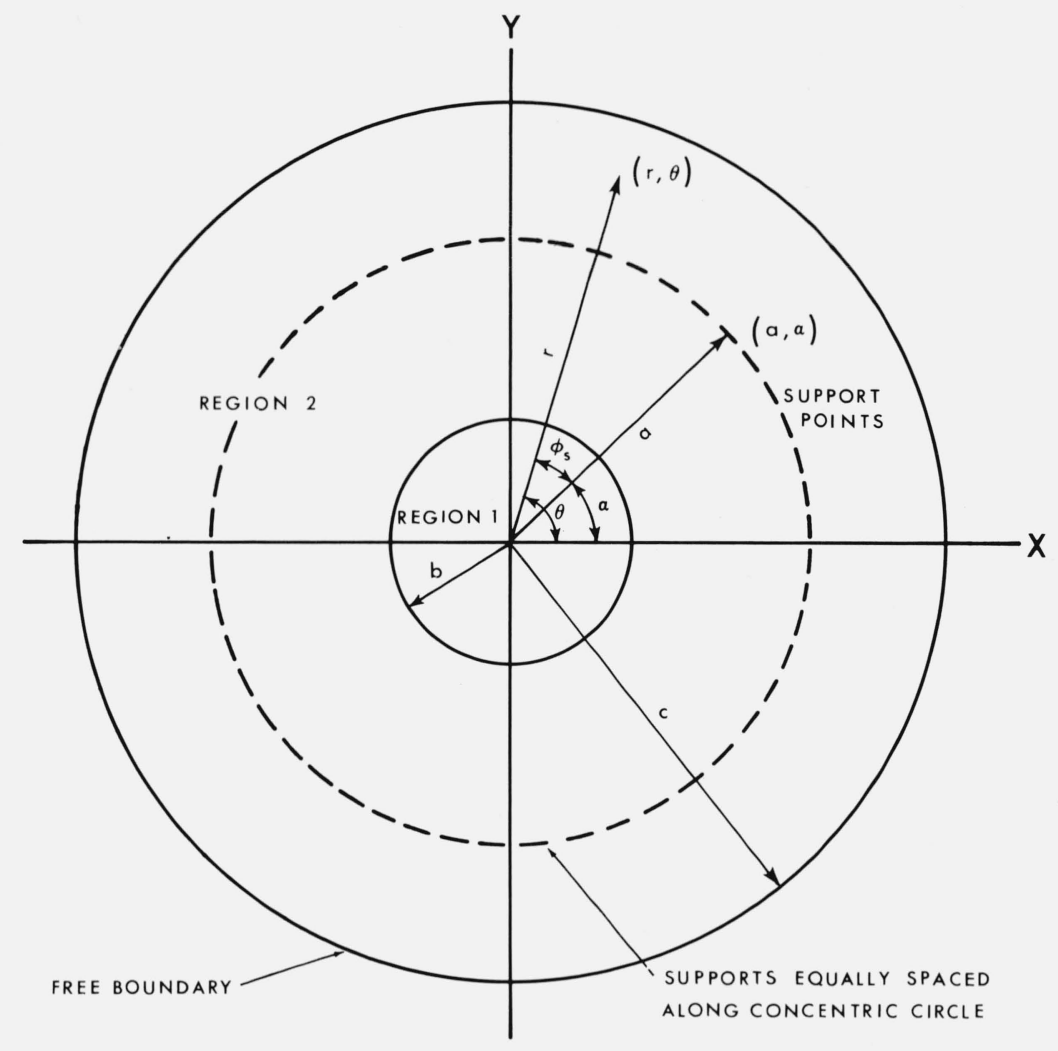

FIGURE 1. Concentric arrangement of the circular plate.

With regard to the preceding discussion it was deemed desirable to extend the previous work [3] to encompass moments, shearing forces, and stresses, for the purpose of presenting equations which may be adapted to the design and analysis of structures of this type.

\section{Moments and Shearing Forces for Symmetrical Bending}

It can be shown that Bassali's solution, reduced to the case of symmetrical bending, yields the following expressions for the radial and tangential bending moments in region 1

$$
M_{r_{1}}^{\prime}=-\frac{P_{0}(1+\nu)}{8 \pi m \kappa}\left[\Psi_{1}^{\prime}\right]+\frac{P_{0}(1-\nu)}{16 \pi m \kappa \rho^{2}}\left[\Omega_{1}^{\prime}\right]
$$

and

$$
M_{t_{1}}^{\prime}=-\frac{P_{0}(1+\nu)}{8 \pi m \kappa}\left[\Psi_{1}^{\prime}\right]-\frac{P_{0}(1-\nu)}{16 \pi m \kappa \rho^{2}}\left[\Omega_{1}^{\prime}\right],
$$

respectively, where

$$
\begin{aligned}
\Psi_{1}^{\prime}=\left[\sum_{s=1}^{m}\left\{\ln \left(1-2 \rho t \cos \varphi_{s}+\rho^{2} t^{2}\right)-\kappa \ln \left(\rho^{2}-2 \rho t \cos \varphi_{s}+t^{2}\right)-\frac{\left(1-t^{2}\right)\left(1-\rho^{2} t^{2}\right)}{\left(1-2 \rho t \cos \varphi_{s}+\rho^{2} t^{2}\right)}\right\}\right. \\
\left.+\frac{2 m t^{2}}{(1+\nu)}+m-\frac{2 m \kappa q^{\prime 2}}{\kappa+1}+2 m \kappa \ln q+\frac{2 m \kappa}{n}\left(\frac{\rho^{n}}{q^{n}}-1\right)\right]
\end{aligned}
$$


and

$$
\begin{aligned}
\Omega_{1}^{\prime}=\left[\sum _ { s = 1 } ^ { m } \left\{\left(\kappa^{2}-1\right) \ln \left(1-2 \rho t \cos \varphi_{s}+\rho^{2} t^{2}\right)+\frac{\left(1-\rho^{2} t^{2}\right)^{2}-2 t^{2}\left(1-\rho^{2}\right)^{2}}{\left(1-2 \rho t \cos \varphi_{s}+\rho^{2} t^{2}\right)}\right.\right. \\
\\
\left.\left.\quad-\frac{\left(1-\rho^{2}\right)\left(1-t^{2}\right)\left(1-\rho^{2} t^{2}\right)^{2}}{\left(1-2 \rho t \cos \varphi_{s}+\rho^{2} t^{2}\right)^{2}}+\frac{\kappa\left(\rho^{2}-t^{2}\right)^{2}}{\left(\rho^{2}-2 \rho t \cos \varphi_{s}+t^{2}\right)}\right\}+m(\kappa-1)\left(\rho^{2}-t^{2}\right)-\frac{4 m \kappa \rho^{n^{\prime}}}{n^{\prime} q^{n}}\right] .
\end{aligned}
$$

For region 2,

$$
M_{r_{2}}^{\prime}=-\frac{P_{0}(1+\nu)}{8 \pi m \kappa}\left[\Psi_{2}^{\prime}\right]+\frac{P_{0}(1-\nu)}{16 \pi m \kappa \rho^{2}}\left[\Omega_{2}^{\prime}\right]
$$

and

$$
M_{t_{2}}^{\prime}=-\frac{P_{0}(1+\nu)}{8 \pi m \kappa}\left[\Psi_{2}^{\prime}\right]-\frac{P_{0}(1-\nu)}{16 \pi m \kappa \rho^{2}}\left[\Omega_{2}^{\prime}\right]
$$

respectively, where

$$
\begin{array}{r}
\Psi_{2}^{\prime}=\left[\sum_{s=1}^{m}\left\{\ln \left(1-2 \rho t \cos \varphi_{s}+\rho^{2} t^{2}\right)-\kappa \ln \left(\rho^{2}-2 \rho t \cos \varphi_{s}+t^{2}\right)-\frac{\left(1-t^{2}\right)\left(1-\rho^{2} t^{2}\right)}{\left(1-2 \rho t \cos \varphi_{s}+\rho^{2} t^{2}\right)}\right\}\right. \\
\left.+\frac{2 m t^{2}}{(1+\nu)}+m-\frac{2 m \kappa q^{\prime 2}}{\kappa+1}+2 m \kappa \ln \rho\right]
\end{array}
$$

and

$$
\begin{gathered}
\Omega_{2}^{\prime}=\left[\sum _ { s = 1 } ^ { m } \left\{\left(\kappa^{2}-1\right) \ln \left(1-2 \rho t \cos \varphi_{s}+\rho^{2} t^{2}\right)+\frac{\left(1-\rho^{2} t^{2}\right)^{2}-2 t^{2}\left(1-\rho^{2}\right)^{2}}{\left(1-2 \rho t \cos \varphi_{s}+\rho^{2} t^{2}\right)}-\frac{\left(1-\rho^{2}\right)\left(1-t^{2}\right)\left(1-\rho^{2} t^{2}\right)^{2}}{\left(1-2 \rho t \cos \varphi_{s}+\rho^{2} t^{2}\right)^{2}}\right.\right. \\
\left.\left.+\frac{\kappa\left(\rho^{2}-t^{2}\right)^{2}}{\left(\rho^{2}-2 \rho t \cos \varphi_{s}+t^{2}\right)}\right\}+m(\kappa-1)\left(\rho^{2}-t^{2}\right)-2 m \kappa \rho^{2}+2 m \kappa q^{\prime 2}\right] .
\end{gathered}
$$

It is noted here that the equation for the twisting moment is the same for regions 1 and 2 and may be expressed as

$$
\begin{gathered}
M_{r t}=\frac{P_{0}(1-\nu)}{8 \pi m \kappa \rho^{2}}\left[\sum _ { s = 1 } ^ { m } \left\{\frac{\rho t \sin \varphi_{s}\left(\rho^{2}-t^{2}\right)}{\left(1-2 \rho t \cos \varphi_{s}+\rho^{2} t^{2}\right)}-\frac{\rho t \sin \varphi_{s}\left(1-\rho^{2}\right)\left(1-t^{2}\right)\left(1-\rho^{2} t^{2}\right)}{\left(1-2 \rho t \cos \varphi_{s}+\rho^{2} t^{2}\right)^{2}}\right.\right. \\
\left.\left.-\frac{\kappa \rho t \sin \varphi_{s}\left(\rho^{2}-t^{2}\right)}{\left(\rho^{2}-2 \rho t \cos \varphi_{s}+t^{2}\right)}-\left(\kappa^{2}-1\right) \arctan \frac{\rho t \sin \varphi_{s}}{1-\rho t \cos \varphi_{s}}\right\}\right]
\end{gathered}
$$

and that the equation for the tangential shearing force is also the same for regions 1 and 2 . The tangential shearing force is given by

$$
Q_{t}=\frac{P_{0}}{4 \pi r m \kappa}\left[\sum_{s=1}^{m}\left\{\frac{2 \kappa \rho t \sin \varphi_{s}}{\left(\rho^{2}-2 \rho t \cos \varphi_{s}+t^{2}\right)}-\frac{2 \rho t \sin \varphi_{s}}{\left(1-2 \rho t \cos \varphi_{s}+\rho^{2} t^{2}\right)}-\frac{2 \rho t \sin \varphi_{s}\left(1-t^{2}\right)\left(1-\rho^{2} t^{2}\right)}{\left(1-2 \rho t \cos \varphi_{s}+\rho^{2} t^{2}\right)^{2}}\right\}\right] .
$$

${ }^{2}$ Bassali's eq (2.44) for $M_{r t_{1}}$ was incorrect in that a factor, his $P_{s}$, was omitted from the summation. No doubt this was a typographical error as his equation for $M_{r t_{2}}$ was correct, and for the general solution of eccentric loading $M_{r t_{1}}$ must equal $M_{r t_{2}}$ at the boundary between regions 1 and 2 . 
The radial shearing forces in regions 1 and 2 as reduced from the general solution are

$$
\begin{aligned}
Q_{r_{1}}^{\prime}=\frac{P_{0}}{4 \pi r m \kappa}\left[\sum _ { s = 1 } ^ { m } \left\{\frac{\kappa\left(\rho^{2}-t^{2}\right)}{\left(\rho^{2}-2 \rho t \cos \varphi_{s}+\rho^{2} t^{2}\right)^{2}}\right.\right. & \frac{\left(1-2 \rho^{2}+\rho^{2} t^{2}\right) t^{2}}{\left(1-2 \rho t \cos \varphi_{s}+\rho^{2} t^{2}\right)} \\
& \left.\left.+\frac{\left(1-t^{2}\right)\left(1-\rho^{2} t^{2}\right)^{2}}{\left(1-2 \rho t \cos \varphi_{s}+\rho^{2} t^{2}\right)^{2}}\right\}+m(\kappa-1)-\frac{2 m \kappa \rho^{2}}{q^{n}}\right]
\end{aligned}
$$

and

$Q_{r_{2}}^{\prime}=\frac{P_{0}}{4 \pi r m \kappa}\left[\sum_{s=1}^{m}\left\{\frac{\kappa\left(\rho^{2}-t^{2}\right)}{\left(\rho^{2}-2 \rho t \cos \varphi_{s}+t^{2}\right)}+\frac{\left(1-2 \rho^{2}+\rho^{2} t^{2}\right) t^{2}}{\left(1-2 \rho t \cos \varphi_{s}+\rho^{2} t^{2}\right)}+\frac{\left(1-t^{2}\right)\left(1-\rho^{2} t^{2}\right)^{2}}{\left(1-2 \rho t \cos \varphi_{s}+\rho^{2} t^{2}\right)^{2}}\right\}-m(\kappa+1)\right]$,

respectively.

\section{The Uniformly Distributed Concentric Load}

The case of the uniformly distributed concentric load with its limiting cases of the concentrated central load and the load uniformly distributed over the entire plate is of more general interest than the case of the symmetrically distributed load given in the preceding section. Therefore this investigation will deal primarily with the case of the uniformly distributed load. It should be noted that no special treatment is required to examine the limiting cases mentioned above, as they are implicit in the solution. The concentrated central load is represented by permitting $q$ to shrink to zero, and the load uniformly distributed over the entire plate is realized by setting $q=1$. It remains then only to select the appropriate equations for the region under analysis.

The equations for moments and shearing forces given in the preceding section were derived for the symmetrically distributed concentric load as defined by eq (1.01). In order to obtain similar equations for the uniformly distributed concentric load it is a simple matter of supplying the appropriate values for $n, n^{\prime}$, and $q^{\prime}$, viz, $n=2, n^{\prime}=4$, and $q^{\prime}=q / \sqrt{2}$. Thus, eqs (1.03) and (1.04) become

$$
M_{r_{1}}=-\frac{P_{0}(1+\nu)}{8 \pi m \kappa}\left[\Psi_{1}\right]+\frac{P_{0}(1-\nu)}{16 \pi m \kappa \rho^{2}}\left[\Omega_{1}\right]
$$

and

$$
M_{t_{1}}=-\frac{P_{0}(1+\nu)}{8 \pi m \kappa}\left[\Psi_{1}\right]-\frac{P_{0}(1-\nu)}{16 \pi m \kappa \rho^{2}}\left[\Omega_{1}\right]
$$

respectively, where eqs (1.05) and (1.06) become

$$
\begin{aligned}
\Psi_{1}=\left[\sum_{s=1}^{m}\left\{\ln \left(1-2 \rho t \cos \varphi_{s}+\rho^{2} t^{2}\right)-\kappa \ln \left(\rho^{2}-2 \rho t \cos \varphi_{s}+t^{2}\right)-\frac{\left(1-t^{2}\right)\left(1-\rho^{2} t^{2}\right)}{\left(1-2 \rho t \cos \varphi_{s}+\rho^{2} t^{2}\right)}\right\}\right. \\
\left.-\frac{2 m t^{2}\left(1+\frac{\kappa q^{2}}{2-t^{2}}\right)}{\kappa+1}+m\left(1+t^{2}\right)+2 m \kappa \ln q+\frac{m \kappa \rho^{2}}{q^{2}}-m \kappa\right]
\end{aligned}
$$

and

$$
\begin{aligned}
\Omega_{1}=\left[\sum _ { s = 1 } ^ { m } \left\{( \kappa ^ { 2 } - 1 ) \operatorname { l n } \left(1-2 \rho t \cos \varphi_{s}\right.\right.\right. & \left.+\rho^{2} t^{2}\right)+\frac{\left(1-\rho^{2} t^{2}\right)^{2}-2 t^{2}\left(1-\rho^{2}\right)^{2}}{\left(1-2 \rho t \cos \varphi_{s}+\rho^{2} t^{2}\right)}-\frac{\left(1-\rho^{2}\right)\left(1-t^{2}\right)\left(1-\rho^{2} t^{2}\right)^{2}}{\left(1-2 \rho t \cos \varphi_{s}+\rho^{2} t^{2}\right)^{2}} \\
& \left.\left.+\frac{\kappa\left(\rho^{2}-t^{2}\right)^{2}}{\left(\rho^{2}-2 \rho t \cos \varphi_{s}+t^{2}\right)}\right\}+m(\kappa-1)\left(\rho^{2}-t^{2}\right)-\frac{m \kappa \rho^{4}}{q^{2}}\right],
\end{aligned}
$$


respectively. Following this same procedure, the radial and tangential bending moments in region 2 , obtained from eqs (1.07) and (1.08) are

and

$$
M_{r_{2}}=-\frac{P_{0}(1+\nu)}{8 \pi m \kappa}\left[\Psi_{2}\right]+\frac{P_{0}(1-\nu)}{16 \pi m \kappa \rho^{2}}\left[\Omega_{2}\right]
$$

$$
M_{t_{2}}=-\frac{P_{0}(1+\nu)}{8 \pi m \kappa}\left[\Psi_{2}\right]-\frac{P_{0}(1-\nu)}{16 \pi m \kappa \rho^{2}}\left[\Omega_{2}\right]
$$

respectively, where eqs (1.09) and (1.10) become

$$
\begin{array}{r}
\Psi_{2}=\left[\sum_{s=1}^{m}\left\{\ln \left(1-2 \rho t \cos \varphi_{s}+\rho^{2} t^{2}\right)-\kappa \ln \left(\rho^{2}-2 \rho t \cos \varphi_{s}+t^{2}\right)-\frac{\left(1-t^{2}\right)\left(1-\rho^{2} t^{2}\right)}{\left(1-2 \rho t \cos \varphi_{s}+\rho^{2} t^{2}\right)}\right\}\right. \\
\left.-\frac{2 m t^{2}\left(1+\frac{\kappa q^{2}}{2 t^{2}}\right)}{\kappa+1}+m\left(1+t^{2}\right)+2 m \kappa \ln \rho\right]
\end{array}
$$

and

$$
\begin{gathered}
\Omega_{2}=\left[\sum _ { s = 1 } ^ { m } \left\{\left(\kappa^{2}-1\right) \ln \left(1-2 \rho t \cos \varphi_{s}+\rho^{2} t^{2}\right)+\frac{\left(1-\rho^{2} t^{2}\right)^{2}-2 t^{2}\left(1-\rho^{2}\right)^{2}}{\left(1-2 \rho t \cos \varphi_{s}+\rho^{2} t^{2}\right)}-\frac{\left(1-\rho^{2}\right)\left(1-t^{2}\right)\left(1-\rho^{2} t^{2}\right)^{2}}{\left(1-2 \rho t \cos \varphi_{s}+\rho^{2} t^{2}\right)^{2}}\right.\right. \\
\left.\left.+\frac{\kappa\left(\rho^{2}-t^{2}\right)^{2}}{\left(\rho^{2}-2 \rho t \cos \varphi_{s}+t^{2}\right)}\right\}+m(\kappa-1)\left(\rho^{2}-t^{2}\right)-2 m \kappa \rho^{2}+m \kappa q^{2}\right]
\end{gathered}
$$

respectively.

Since the expressions for the twisting moment, $M_{r t}$, the tangential shearing force, $Q_{t}$, and the radial shearing force in region $2, Q_{r_{2}}^{\prime}$ are independent of the load distribution, eqs (1.11), (1.12), and (1.14) are also appropriate for the case of the uniformly distributed load. However, $Q_{r_{1}}^{\prime}$ of eq (1.13) being dependent on $n$, becomes

$$
\begin{aligned}
Q_{r_{1}}^{\prime}=\frac{P_{0}}{4 \pi r m \kappa}\left[\sum _ { s = 1 } ^ { m } \left\{\frac{\kappa\left(\rho^{2}-t^{2}\right)}{\left(\rho^{2}-2 \rho t \cos \varphi_{s}+t^{2}\right)}+\right.\right. & \frac{\left(1-2 \rho^{2}+\rho^{2} t^{2}\right) t^{2}}{\left(1-2 \rho t \cos \varphi_{s}+\rho^{2} t^{2}\right)} \\
& \left.\left.+\frac{\left(1-t^{2}\right)\left(1-\rho^{2} t^{2}\right)^{2}}{\left(1-2 \rho t \cos \varphi_{s}+\rho^{2} t^{2}\right)^{2}}\right\}+m(\kappa-1)-\frac{2 m \kappa \rho^{2}}{q^{2}}\right]
\end{aligned}
$$

for uniform loading.

\section{Stress at the Center of the Plate}

An examination of eq (1.11) shows that $M_{r t}=0$ as a limit when $\rho \rightarrow 0$, and is independent of the angular orientation. Therefore Mohr's circle becomes a point, $M_{t_{1}}=M_{r_{1}}$, and $\sigma_{r_{1}}=\sigma_{t_{1}}$. It is noted that $M_{r_{1}}$ and $M_{t_{1}}$ given in eqs (1.15) and (1.16), respectively, differ only in the sign of the second term of each equation, and that these terms, having $\rho^{2}$ in the denominator cannot be solved directly when $\rho=0$. However, they can be evaluated by setting $\boldsymbol{M}_{r_{1}}=M_{t_{1}}$. Thus, eqs (1.15) and (1.16) yield

$$
\begin{gathered}
{\left[\sum _ { s = 1 } ^ { m } \left\{\left(\kappa^{2}-1\right) \ln \left(1-2 \rho t \cos \varphi_{s}+\rho^{2} t^{2}\right)+\frac{\left(1-\rho^{2} t^{2}\right)^{2}-2 t^{2}\left(1-\rho^{2}\right)}{\left(1-2 \rho t \cos \varphi_{s}+\rho^{2} t^{2}\right)}-\frac{\left(1-\rho^{2}\right)\left(1-t^{2}\right)\left(1-\rho^{2} t^{2}\right)^{2}}{\left(1-2 \rho t \cos \varphi_{s}+\rho^{2} t^{2}\right)^{2}}\right.\right.} \\
\left.\left.+\frac{\kappa\left(\rho^{2}-t^{2}\right)^{2}}{\left(\rho^{2}-2 \rho t \cos \varphi_{s}+t^{2}\right)}\right\}+m(\kappa-1)\left(\rho^{2}-t^{2}\right)-\frac{m \kappa \rho^{4}}{q^{2}}\right]=0
\end{gathered}
$$


from which it is obvious that the second terms of eqs (1.15) and (1.16) are equal to zero when $\rho=0$. The moments at the center of the plate $(\rho=0)$ may now be written as

$$
M_{r_{1}}=M_{t_{1}}=-\frac{P_{0}(1+\nu)}{8 \pi m \kappa}\left[-2 m \kappa \ln t+\frac{2 m \kappa t^{2}\left(1-\frac{q^{2}}{2 t^{2}}\right)}{\kappa+1}+2 m \kappa \ln q-m \kappa\right]_{\rho=0}
$$

and the stresses on the surface are given by

$$
\sigma_{r_{1}}=\sigma_{t_{1}}= \pm \frac{3 P_{0}(1+\nu)}{4 \pi h^{2}}\left[\frac{2 t^{2}\left(1-\frac{q^{2}}{2 t^{2}}\right)}{\kappa+1}+2 \ln \frac{q}{t}-1\right]_{\rho=0} .
$$

As a matter of convenience for graphical representation eq (1.25) may be written as

$$
\sigma_{r_{1}}=\sigma_{t_{1}}=-\frac{3 P_{0}}{4 \pi h^{2}}[\xi-\lambda]
$$

where

$$
\begin{aligned}
& \xi=(1+\nu) \ln q^{2}+\frac{(1-\nu)}{2} q^{2} \\
& \lambda=(1+\nu)\left(1+\ln t^{2}\right)+(1-\nu) t^{2} .
\end{aligned}
$$

Figure 2 is the graphical representation of eqs (1.27) and (1.28) for $\nu=0.3$. It is apparent from the $\lambda$ curve that the stresses at the center of the plate are also affected by the portion of the plate that overhangs the supports. The $\xi$ curve shows how $q$ affects the stresses at the center. It should be noted that when $q \rightarrow 0, \sigma_{r}$ and $\sigma_{t} \rightarrow \infty$. It is obvious that this theory cannot be used to compute stress at or very near a concentrated load or reaction point.

An examination of eq (1.25) shows that the stresses at the center of the plate are independent of $m$ and $\theta$, and it can be shown that this equation reduces to

$$
\sigma=\frac{3 P_{0}}{8 \pi h^{2}}(3+\nu)
$$

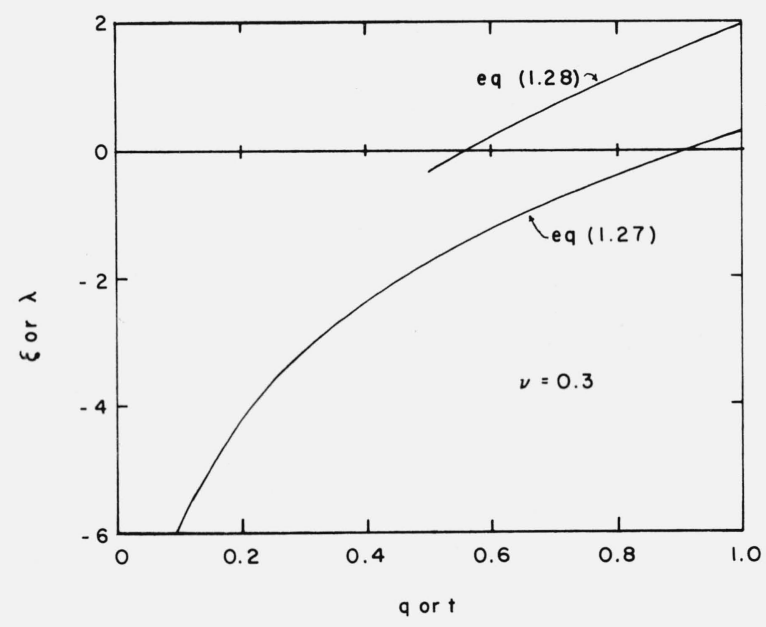

FIGURE 2. Factors which affect the stress at the center of the plate. 


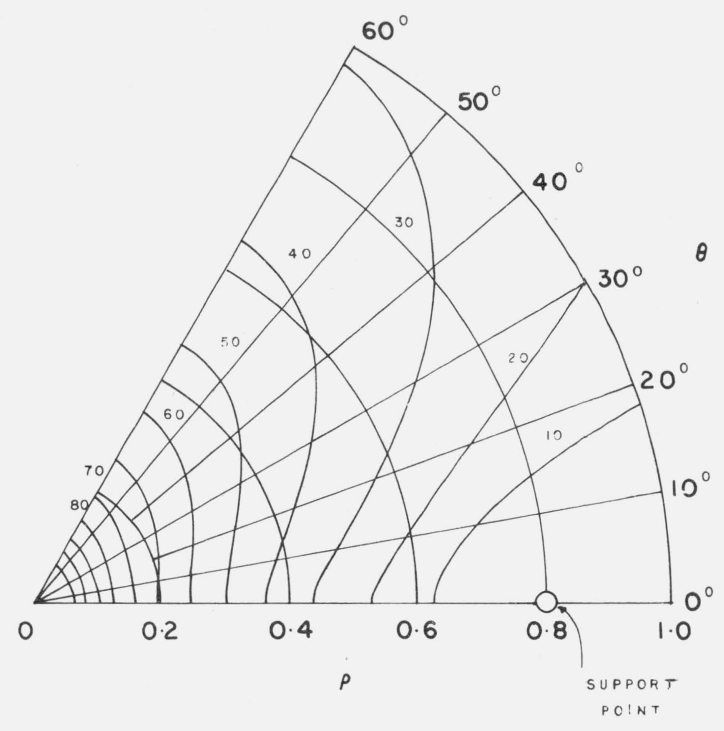

FIGURE 3. Distribution of maximum principal stresses in the plate.

for the case of a simply supported, uniformly loaded plate $(t=q=1)$. Furthermore, for the case of a simply supported circular plate having a central load $(q=b / c$ and $t=1)$ eq (1.25) reduces to

$$
\sigma=\frac{3 P_{0}(1+\nu)}{2 \pi h^{2}}\left[\frac{1}{(1+\nu)}+\ln \frac{c}{b}-\frac{b^{2}}{4 c^{2}} \frac{(1-\nu)}{(1+\nu)}\right] .
$$

Thus, it is shown that for these limiting cases eq (1.25) reduces to the Grashof equations.

It should be noted that the usable strength of a plate is usually greater than that implied by eqs (1.25), (1.26), (1.29), and (1.30) [4]. These are elastic equations, and experience shows that local yielding of materials at a highly stressed point does not necessarily indicate structural failure or excessive deflection. Therefore, predictions of structural failure based on these equations would require the use of an appropriate theory of failure.

Moments and Stresses Elsewhere in the Plate. To illustrate the stress distribution over a segment of a plate, figure 3 presents lines of equal maximum principal stress sensitivity $\left(\sigma / P_{0}\right)$ over one half the period of symmetrical distribution. This figure was prepared from the results obtained from eqs (1.11),(1.19), and (1.20) for the following conditions: $m=3, t=0.8, q=0, \nu=0.3$, and $h=0.125$. It can be noted from the figure that the support is located on the ray $\theta=0^{\circ}$, and that the larger values of the maximum principal stress sensitivity extend the farthest out from the center along the ray $\theta=\pi / m$. These rays have further distinction in that an examination of the twisting moment, eq (1.11), shows that $M_{r t}=0$ for any values of $\rho$ and $t$ along these angular orientations. It follows then that the bending moments, $M_{r}$ and $M_{t}$, along these rays produce principal stresses.

\section{Comparison of Theoretical and Experimental Results}

The test results presented herein were obtained from retesting specimen A of a previous investigation [3]. Since the specimens, materials, and test methods were fully described in the previous paper, it suffices here to indicate that the dead load method of testing was used, and the tangential strains were measured along the support circle $(t=0.976$ and $m=3)$ by means of foil type electrical resistance strain gages having a gage length of $1 / 8 \mathrm{in}$. These gages were mounted on the top and bottom surface of the specimen at the angular locations indicated in figure 4 . 


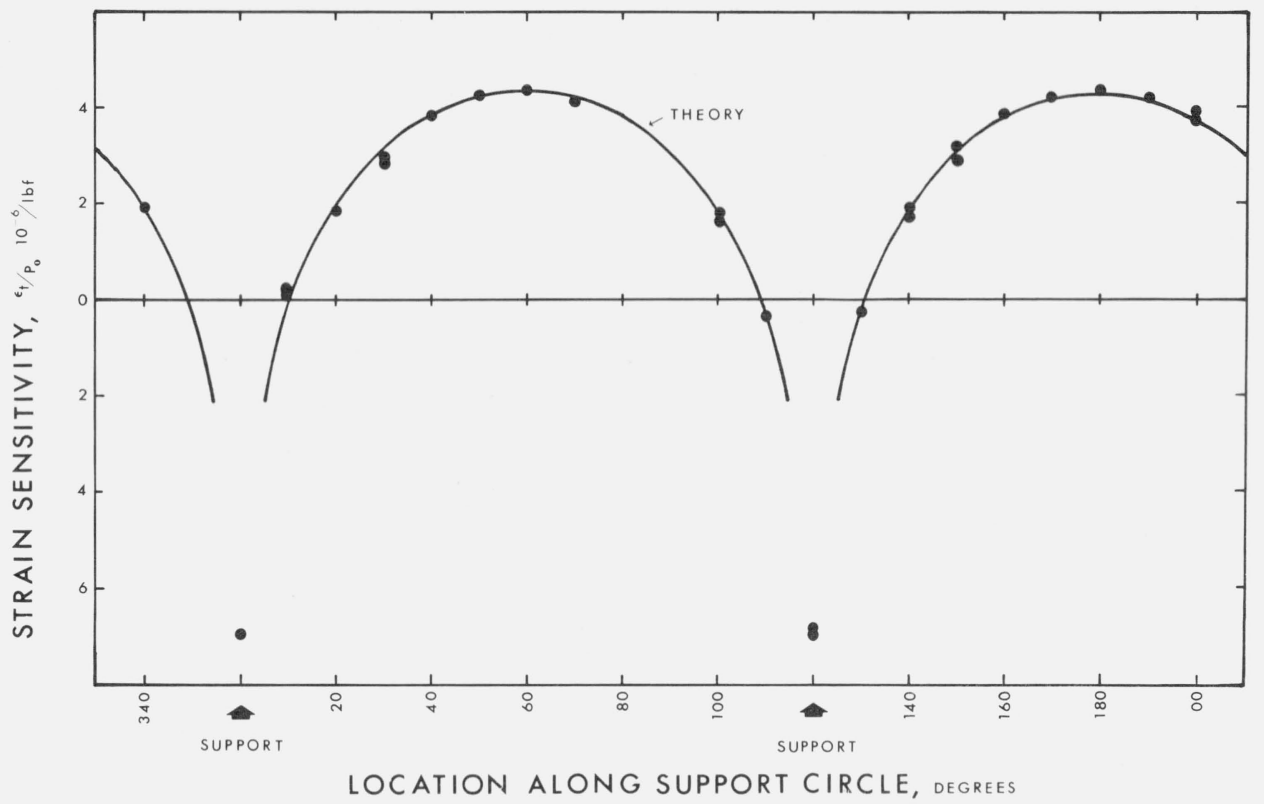

FIGURE 4. Theoretical and experimental strain sensitivities along the support circle.

The tangential strains were computed for the expression

$$
\epsilon_{t_{2}}=\frac{1}{E}\left(\sigma_{t_{2}}-\nu \sigma_{r_{2}}\right)= \pm \frac{6}{E h^{2}}\left(M_{t_{2}}-\nu M_{r_{2}}\right) .
$$

Inserting the appropriate values for radial and tangential bending moment from eqs (1.19) and (1.20) yields

$$
\epsilon_{t_{2}}= \pm \frac{3 P_{0}\left(1-\nu^{2}\right)}{4 \pi m E h^{2} \kappa}\left(\Psi_{2}+\frac{\Omega_{2}}{2 \rho^{2}}\right)
$$

where $\Psi_{2}$ and $\Omega_{2}$ are given by eqs (1.21) and (1.22), respectively.

The agreement between the experimental data, which represents two separate sets of test results, and the theoretical results shown in figure 4 is very good. This, along with the good agreement reported for deflection [3], lends credence to the use of the theory to predict the flexural behavior of the plates.

\section{Summary}

The special application of Bassali's more general solution presented herein provides the equations necessary to calculate the elastic moments, shearing forces, and stresses anywhere in a thin circular elastic plate supported at points equally spaced along a concentric support circle and subjected to symmetrical bending. However, the maximum stresses at the center of the plate are probably of more general interest in the design and analysis of the thin circular plate subjected to a uniformly distributed concentric load. It is interesting to note that the equations take on a very simple form for these stresses and appear to be well adapted for design purposes. It is of 
further interest that these stresses, eq (1.25), are independent of angular orientation and the number of supports. As in the case of deflection [3], the effect of the annular portion of the plate overhanging the support circle is evident as illustrated by the $\lambda$ curve in figure 2 .

Caution should be exercised in the use of these elastic equations for design purposes as local yielding may not be a valid design criterion for the particular material and structure under consideration. The selection of an appropriate theory of failure is of the utmost importance in this case.

The good agreement between the theoretical and experimental strains presented herein along with that for deflection [3] serve to substantiate the ability of the theory to predict the flexual behavior of the plates.

The authors are indebted to W. H. Pell of the National Science Foundation for his advice during the initial phases of this investigation, and to D. R. Tate for his valuable suggestions. Special mention is due L. J. Davis for his active participation in the Laboratory.

\section{References}

[1] Bassali, W. A., The Transverse Flexure of Thin Elastic Plates Supported at Several Points, Proceedings Cambridge Philosophical Society, Vol. 53, 728-743, 1957.

[2] Morley, A., Strength of Materials (Longmans, Green and Company, 8th ed., 1935).

[3] Kirstein, A. F., W. H. Pell, R. M. Woolley, and L. J. Davis, Deflection of centrally loaded thin circular elastic plates on equally spaced point supports, J. Res. NBS 70C (Engr. and Instr.) No. 4, 227 (1966).

[4] Seely, F. B., Advanced Mechanics of Materials (John Wiley \& Sons, Inc., New York, N.Y., First Edition, 1950).

(Paper 71C1-438) 